\title{
FIRST ORDER DIFFERENTIAL CLOSURES OF CERTAIN PARTIALLY ORDERED FIELDS
}

\author{
BY
}

\author{
JOSEPH E. TURCHECK
}

ABSTRACT. First order algebraic differential equations (a.d.e.'s) are considered in the setting of an abstract differential field with an abstract order relation, whose properties mirror those of the usual asymptotic dominance relations of analysis. An abstract existence theorem, for such equations, is proved by constructing an extension of both the differential field and the abstract order relation. As a consequence, a first order differential closure theorem, for those differential fields with order relations which we consider, is obtained. The closure theorem has corollaries which are important to the asymptotic theory of a.d.e.'s and have application to a.d.e.'s with coefficients meromorphic in a sector of the complex plane.

Introduction. This paper is divided into six parts.

I. Preliminaries.

II. First order differential closure theorem.

III. First orderenth degree closed GLF's.

IV. Abstract existence theorem.

V. Applications.

VI. Appendix.

Let $\mathbf{Z}=((K, \ll, U, C), D, \mathbf{x})$ be a graduated logarithmic field (GLF), as defined by Strodt [4], also [6]. In this paper we make the assumptions that $C$ is an algebraically closed field and, moreover, that, whenever $B$ is a finite subset of $K, \exists$ a field, constrained over $Z$, which contains $B$. A GLF with the latter property will be said to be finitely enveloped. (Cf. [6] for any undefined terms used in this paper.)(1) It might be remarked that the simplest example of such a GLF is the differential field of functions generated by the logarithmic monomials

Presented to the Society, April 21, 1973; received by the editors June 19, 1973. AMS (MOS) subject classifications (1970). Primary 34E05, 34A20, 12H05.

Key words and phrases. Abstract existence theorem, al gebraic differential equation, asymptotically nonsingular, asymptotic order relation, attached, critical chain, critical monomial, critically unique, differential closure property, differential field extension, differential polynomial, finitely enveloped, graduated logarithmic field, logarithmic monomial, principal monomial, rank stable, stability of a differential polynomial, structure theorem, valuation.

(1) With the exception that $f \geq g$ means that either $f \gg g$ or $f \approx g$, where $\gg$ and $\approx$ are as defined in [6], and, similarly, for $f \leq g$. 
(i.e., functions of a complex variable of the form, $c z^{a}(\log z)^{b} \ldots\left(\log _{r} z\right)^{t}$, where $c$ is a complex number, $a, b, \cdots, t$ are rational, $r$ is a nonnegative integer, and $\log _{r} z$ is the $r$ th iterate of the logarithm). In this example, $(K, D)$ is the differential field described above, $\ll$ is the usual $\left({ }^{2}\right)$ asymptotic ordering of functions as $z \rightarrow \infty$ in a sector of the complex plane, $U$ consists of all unit logarithmic monomials, i.e., those monomials with $c=1, C$ is the complex number field and $x$ is the sequence $\left(z, \log z, \log _{2} z, \ldots\right)$.

We note a basic fact: $\cdot$ If $R$ is a differential polynomial (d.p.) over $\mathbf{Z}$ and $f \in K$ is a nonzero solution of $R=0$, then $R$ is unstable at $f$. Moreover, if $m$ is the unique logarithmic monomial $\sim f$, then $m \in$ crit $(R, Z)$.

Does there exist a differential field extension of $(K, D)$, say $\left(K^{*}, D^{*}\right)$, and an extension of $\ll$, the asymptotic order (cf. $[3, \S 1]$, also $[6, \S 2.3]$ ) of $K$, to an asymptotic order of $K^{*}$, say $\ll^{*}$, such that $Z^{*}=\left(\left(K^{*},<^{*}, U, C\right), D^{*}, \mathbf{x}\right)$ is a finitely enveloped GLF with the closure property: Whenever $P$ is a d.p. over $\mathbf{Z}^{*}$ and $m \in \operatorname{crit}\left(P, \mathbf{Z}^{*}\right), \exists$ an element of $K^{*}$, say $b$, such that $b \sim^{*} m$ and $P(b)=0$ ?

The main result of this paper is the solution of the above extension problem when the closure property is taken with respect to (w.r.t.) first order d.p.'s.

We require $C$ to be algebraically closed and $\mathbf{Z}$ (also $\mathbf{Z}^{*}$ ) to be finitely enveloped because then Bank's algorithm (cf. [1], also $[6, \S \$ 2.81-85]$ ) can be effectively applied to any d.p. over $\mathbf{Z}$, say $P$, to determine crit $(P, \mathbf{Z})$, the set of P's critical monomials.

After preliminary work in I, the first order differential closure theorem is stated in II and reduced there, by a Zorn's lemma argument, to the abstract existence theorem. III contains some results which hold for GLF's having the closure property w.r.t. certain classes of d.p.'s. The arguments of III were developed by Strodt in order to prove the results announced in [5].

The abstract existence theorem is proved in IV. Briefly, an appropriate d.p. over $\mathbf{Z}$, say $Q$, is selected. A proper differential field extension of $(K, D)$, say $\left(K^{\prime}, D^{\prime}\right)$, which is generated over $(K, D)$ by a solution of $Q=0$ is constructed. « is extended to an asymptotic order of $K^{\prime}$, say $<^{\prime}$. Standard facts from algebra and differential algebra about field extensions are used to construct $\left(K^{\prime}, D^{\prime}\right)$ while the concept of a valuation (cf. [2, pp. 296-300]) is used to define $<^{\prime}$. It is then necessary to show that $Z^{\prime}=\left(\left(K^{\prime},<^{\prime}, U, C\right), D^{\prime}, x\right)$ is a GLF. The major difficulty here is to verify that $D^{\prime}$ is stable, in the context of $Z^{\prime}$, at nonconstant logarithmic monomials (cf. $[6, \$ 2.4(2)]$ ). The preliminary "structure theorem" of III is crucial for this. Indeed, only a certain inductive point of view in the selection of $Q$ will enable us to use this theorem to advantage. Roughly, if $n$

(2) Al though, for this example, $f \ll g$ coincides with $f=o(g)$, generally $f \ll g$ is much stronger than $f=o(g)$ (cf. $[6, \S 2.121 \mathrm{D}]$ ). 
is the maximal integer such that $\mathbf{Z}$ has the closure property w.r.t. first order d.p.'s of degree $\leq n$, then we take $Q$ to be first order and $(n+1)$ st degree.

Applications of the first order differential closure theorem are given in V. The applications hold in any (finitely enveloped or not) GLF, abstract or concrete (i.e., function-theoretic). (Cf. $[6, \$ \$ 2.120-122]$ for examples of concrete GLF's.) Our main application is the establishment of a general "first order structure theorem". Roughly, a first order structure theorem denies to a first order a.d.e. the possibility of possessing an infinite sequence of solutions, $v_{0}, v_{0}+$ $v_{1}, \cdots, v_{0}+v_{1}+\cdots+v_{n}, \cdots$, such that $v_{i+1} \ll v_{i} \forall i$. Earlier versions of the structure theorem have proved extremely useful in the algebraic-asymptotic theory of differential equations (cf. [6, Chapter 6]). Our structure theorem, the strongest one so far, generalizes a recent result of Strodt [5].

VI is an appendix containing results which would be disruptive if placed elsewhere.

The author gratefully acknowledges the invaluable advice and support of Walter Strodt and several informative conversations with Robert Wright. In particular, the important concept of a "P-stable element" (cf. \$4.7.D) is due to Wright.

Notation. $r$ will denote the nonnegative integers. In this paper the letter $D$ (L) when suffixed to a section number will indicate a section devoted to a definition (lemma).

General hypothesis for I-IV. $\mathrm{Z}=((K, \ll, U, C), D, \mathbf{x})$ is a finitely enveloped GLF and $C$ is algebraically closed. $M(U)$ will denote the set of (unit) logarithmic monomials of $\mathbf{Z}$.

I. Preliminaries.

General hypothesis for $\mathbf{I} . Z^{\prime}=\left(\left(K^{\prime},<^{\prime}, U, C\right), D^{\prime}, \mathbf{x}\right)$ is a finitely enveloped GLF. $\left(K^{\prime}, D^{\prime}\right)$ and $\ll^{\prime}$ are extensions, respectively, of $(K, D)$ and $\ll$.

1.1. D. $f \in K^{\prime}$ is said to be nonsingular over $K$ if either (1) $f$ is algebraic over $K$, or (2) $f$ satisfies a first order a.d.e. over $(K, D)$, say $P=0$, and $P$ is asymptotically nonsingular at $f$.

1.2. D. $Z^{\prime}$ is said to be strongly attached to $Z$ if $K^{\prime}$ is differentially generated over $K$ by its nonsingular elements over $K$.

1.3. D. $Z^{\prime}$ is said to be weakly attached to $Z$ if, whenever $f \in K^{\prime}, \exists$ a finite, nondecreasing sequence of differential subfields of $K^{\prime}$, say $K=K_{0}, K_{1}$, $\cdots, K_{n}$, such that (1) if $\mathbf{Z}=Z_{0}, Z_{1}, \ldots, Z_{n}$ is the sequence of GLF's associated to the $K_{i}$ in the obvious way, then $\mathbf{Z}_{i+1}$ is strongly attached to $\mathbf{Z}_{i}(0$ $\leq i \leq n-1)$ and (2) $f \in K_{n}$.

1.4. L. Suppose that $\mathrm{Z}^{\prime \prime}$ is strongly attached to $\mathrm{Z}^{\prime}$ and $\mathrm{Z}^{\prime}$ is weakly attacbed to $\mathbf{Z}$. Then $\mathbf{Z}^{\prime \prime}$ is weakly attached to $\mathbf{Z}$. 
Proof. This follows from the definitions.

\subsection{If $\mathbf{Z}^{\prime}$ is strongly attached to $\mathrm{Z}$, then $\operatorname{card}(K)=\operatorname{card}\left(K^{\prime}\right)$.}

Proof. Let $G=\left\{f \in K^{\prime}: f\right.$ is nonsingular, but not algebraic over $\left.K\right\}$. $K^{\prime}$ is clearly algebraic over $K(G)$. Thus, the transcendence degree of $K^{\prime}$ over $K$ is $\leq \operatorname{card}(G)$. By a well-known cardinality result, card $\left(K^{\prime}\right)>\operatorname{card}(K)$ iff the transcendence degree of $K^{\prime}$ over $K$ is $>$ card $(K)$. It suffices to show that $\operatorname{card}(G)>$ $\operatorname{card}(K)$ leads to a contradiction.

If $f \in G, \exists$ a first order d.p. over $Z$, say $Q$, and $q \in$ crit $(Q, Z)$ such that $f \sim q, Q(f)=0$, and $Q$ is asymptotically nonsingular at $f$. Under the above circumstances, we say that $f$ is associated to $(Q, q)$. Let $L$ denote the set $\{(P, m): f$ is associated to $(P, m)$ for some $f \in G\}$; clearly card $(L) \leq \operatorname{card}(K)$. Thus, since card $(G)>\operatorname{card}(K), \exists$ an element of $L$, say $(P, m)$, whose set of associates, say $H$, has cardinality $>$ card $(K)$.

Select $b_{0} \in H$. Then $P\left(b_{0}\right)=0$. Let $P_{1}(Y)=P\left(b_{0}+Y\right)$ and $H_{1}=\left\{f-b_{0}\right.$ : $\left.f \in H, f \neq b_{0}\right\}$ and $C_{1}=\left\{p \in \operatorname{crit}\left(P_{1}, \mathrm{Z}\right): p<<b_{0}\right\}$. Then, clearly, card $\left(H_{1}\right)>$ $\operatorname{card}(K)$ and $\operatorname{card}\left(C_{1}\right) \leq \operatorname{card}(K)$. Each element of $H_{1}$ is $\sim$ to some element of $C_{1}$. Thus $\exists q \in C_{1}$ such that $\operatorname{card}\left(\left\{f \in H_{1}: f \sim q\right\}\right)>\operatorname{card}(K)$. Select $b_{1} \in H_{1}$ such that $b_{1} \sim q$. Then $P_{1}\left(b_{1}\right)=0$. Let $P_{2}(Y)=P_{1}\left(b_{1}+Y\right)$ and $H_{2}=\left\{f-b_{1}\right.$ : $f \sim q$ and $\left.f \in H_{1}-\left\{b_{1}\right\}\right\}$ and $C_{2}=\left\{p \in \operatorname{crit}\left(P_{2}, Z\right): p<<b_{1}\right\}$. Then, as before $\operatorname{card}\left(H_{2}\right)>\operatorname{card}(K)$ and $\operatorname{card}\left(C_{2}\right) \leq \operatorname{card}(K)$. At this point, we may proceed as above, with $P_{2}, H_{2}$, and $C_{2}$ replacing $P_{1}, H_{1}$, and $C_{1}$. From this recursive procedure, we infer the existence of an infinite sequence into $K^{\prime}$, say $b_{0}, b_{1}, b_{2}$, $\cdots$, such that $b_{i+1} \ll b_{i}$ and $P\left(b_{0}+b_{1}+\cdots+b_{i}\right)=0 \forall i \geq 0$. Since $P$ is asymptotically nonsingular at $m$, the structure theorem of $[6, \$ 6.10]$ is contradicted.

Proof. This is a rather straightforward extension of $\$ 1.5$.

1.7. D. (a) Let $F$ be a family of d.p.'s over $Z$. Then $Z$ is said to be closed w.r.t. $F$ iff, whenever $P \in F$ and $m \in \operatorname{crit}(P, \mathrm{Z}), P=0$ has a solution $\sim m$ in $K$.

(b) Let $n$ be a positive integer. Let $G$ be the set of all polynomials over $K$. Let $H$ be the set of all first order d.p.'s over $Z$ and $L$ the set of all elements of $H$ whose degree w.r.t. $D Y$ is $\leq n$. Then we say $\mathrm{Z}$ is:

(i) algebraically closed iff it is closed w.r.t. $G$,

(ii) first order closed iff it is closed w.r.t. $G \cup H$,

(iii) first order-nth degree closed iff it is closed w.r.t. $G \cup L$.

1.6. L. If $\mathrm{Z}^{\prime}$ is weakly attached to $\mathrm{Z}$, then $\operatorname{card}\left(K^{\prime}\right)=\operatorname{card}(K)$.

II. First order differential closure theorem. 
General hypothesis for II. $G$ is an algebraically closed field extension of $K$ and $\operatorname{card}(G)>\operatorname{card}(K)$.

Abstract existence theorem. Suppose that $\mathbf{Z}$ is not first order closed. Then 3 a proper differential algebraic extension of $(K, D)$, say $\left(K^{\prime}, D^{\prime}\right)$, and an extension of $\ll$, say $\ll^{\prime}$, such that $K^{\prime} \subset G$ and $Z^{\prime}=\left(\left(K^{\prime},<^{\prime}, U, C\right), D^{\prime}, \mathbf{x}\right)$ is a finitely enveloped $G L F$ which is strongly attached to $\mathbf{Z}$.

Proof. The proof is given in IV.

First order differential closure theorem. Suppose that $\mathbf{Z}$ is not first order closed. Then $\exists$ a differential algebraic extension of $(K, D)$, say $\left(K^{*}, D^{*}\right)$, and an extension of $\ll$, say $\ll^{*}$, such that $Z^{*}=\left(\left(K^{*},<^{*}, U, C\right), D^{*}, \mathbf{x}\right)$ is a first order closed GLF and weakly attacbed to $\mathrm{Z}$. $\mathrm{Z}^{*}$ will be called a first order closure of $\mathbf{Z}$.

Proof. Let $\mathcal{F}$ be the family of all ordered triples, $\left(K_{i}, D_{i}, \ll<_{i}\right)$, such that (1) $K_{i} \subset G$, (2) $\left(K_{i}, D_{i}\right)$ is an extension of $(K, D),(3)<_{i}$ is an extension of $\ll$, and (4) $\left(\left(K_{i}, \ll<_{i}, U, C\right), D_{i}, \mathbf{x}\right)$ is a finitely enveloped GLF which is weakly attached to $Z$. We order $\mathcal{F}$ as follows: $\left(K_{i}, D_{i}, \ll<_{i}\right)>\left(K_{j}, D_{j}, \ll<_{j}\right)$ iff $\left(K_{i}, D_{i}\right)$ is an extension of $\left(K_{j}, D_{j}\right)$ and $\ll_{i}$ is an extension of $\ll_{j}$. It follows routinely from the definitions of $\mathrm{Part} I$ that $\mathscr{F}$ is thus inductively ordered. Let $\left(K^{*}, D^{*}\right.$, $\left.<^{*}\right)$ be a maximal element of $\mathcal{F}$, as guaranteed by Zorn's lemma. $Z^{*}=\left(\left(K^{*}, \ll *\right.\right.$, $\left.U, C), D^{*}, \mathbf{x}\right)$ is first order closed since in the contrary case the abstract existence theorem together with $\S 1.4$ and $\$ 1.6$ provide a contradiction to the maximality of $\left(K^{*}, D^{*},<^{*}\right)$.

\section{First order-nth degree closed GLF's.}

General hypothesis for III. $n$ is a positive integer. $Z$ is first order-nth degree closed. $P$ is either a first order d.p. over $\mathrm{Z}$ of degree $\leq n$ or a zero order d.p.

3.1. D. Let $\mathrm{u}=\left(u_{0}, u_{1}, \cdots\right)$ be a sequence into $K$ such that $u_{i+1} \ll u_{i}$ $\forall i \geq 0$. Let $P_{0}=P$ and $P_{i+1}=P_{i}\left(u_{i}+Y\right) \forall i \geq 0$. Let $f \in K$.

(1) $\mathrm{u}$ is a critical chain for $P$ iff $P_{i}$ is unstable at $u_{i} \forall i \geq 0$.

(2) $f \approx \Sigma(\mathrm{u}, k)$ means $f-\left(u_{0}+\cdots+u_{i}\right) \ll u_{i}$ holds whenever $0 \leq i \leq k$.

(3) $f \approx \Sigma(\mathrm{u})$ means $f \approx \Sigma(\mathrm{u}, k)$ holds $\forall k \geq 0$.

3.2. L. Let $\mathbf{u}$ be a critical chain for $P$. Suppose that $P$ is either asymptotically nonsingular at $u_{0}$ or zero order. Then $P=0$ bas a solution in $K$, say f. sucb that $f \approx \Sigma(\mathrm{u})$.

Proof. Let $P_{i}$ be defined as in \$3.1. For each $i$, let $z_{i} \in K$ be a solution of $P_{i}=0$ which is $\sim u_{i}$. Moreover, let $y_{0}=z_{0}$ and $y_{i+1}=u_{0}+u_{1}+\cdots+u_{i}+$ $z_{i+1}$ for $i \geq 0$. We claim that, for some $j, y_{j} \approx \Sigma(u)$. 
Suppose the contrary. For each $j$, let $q_{j}$ be the smallest integer such that $y_{j} \approx \Sigma\left(\mathrm{u}, q_{j}\right)$. Clearly, $q_{j} \geq j \forall j \geq 0$. Thus $\mathbf{q}=\left(q_{0}, q_{1}, \ldots\right)$ is an unbounded sequence. W.1.o.g., we assume that $q$ is strictly increasing. For each $j$, let $w_{j}=y_{j}-\left(u_{0}+\cdots+u_{q_{j}}\right)$. Clearly, $w_{j} \geq u_{q_{j}} \forall j \geq 0$. But, since $y_{j} \approx$ $\Sigma\left(\mathrm{u},-1+q_{j}\right)$ and $u_{q_{j}}<<u_{-1+q_{j}}$, we have $w_{j} \ll u_{-1+q_{j}} \forall j \geq 1$. It follows easily that $w_{j+1} \ll w_{j} \forall j \geq 0$. Let $v_{0}=y_{0}$ and $v_{j+1}=y_{j+1}-y_{j} \forall j \geq 0$. Thus, $v_{j+1}=$ $w_{j+1}-w_{j}+\left(u_{1+q_{j}}+\cdots+u_{q_{j+1}}\right)$. Clearly, $v_{j+1} \sim-w_{j} \forall j \geq 0$. Thus, $v_{j+1}<<v_{j}$ $\forall j \geq 0$. Therefore $P\left(v_{0}+v_{1}+\cdots+v_{j}\right)=0 \forall j \geq 0$. If $P$ is asymptotically nonsingular at $u_{0}, \$ 6.10$ of $[6]$ is contradicted. On the other hand, the contradiction is obvious if $P$ is zero order.

3.3. L. Let $\mathbf{u}$ be a critical chain for $P$. Then $P=0$ bas a solution in $K$, say $f$, sucb that $f \approx \Sigma(u)$.

Proof. Consider $S \mathscr{S}\left(P, u_{0}\right)$ (cf. $[6, \$ 6.0]$ for teminology). If $S \mathscr{S}\left(P, u_{0}\right)=0$, the result follows from \$3.2. Assume this lemma is true for $\mathscr{S}\left(P, u_{0}\right) \leq s$ and suppose $S \mathscr{S}\left(P, u_{0}\right)=s+1$.

Let $P_{i}$ be defined as in $\$ 3.1$. If $S \mathscr{S}\left(P_{j}, u_{j}\right)<s+1$ for some $j>0$, the result follows from the induction hypothesis. Thus, we assume $S_{S}\left(P_{j}, u_{j}\right)=s+1 \forall j \geq$ 0 . Let $Q=\Gamma(P), Q_{0}=Q$, and $Q_{i+1}=Q_{i}\left(u_{i}+Y\right) \forall i \geq 0$. u is a critical chain for $Q$. Thus, by the induction hypothesis, $\exists g \in K$ such that $Q(g)=0$ and $g \approx \Sigma(u)$.

Let $R=P(g+Y)$. If $R(0)=0$, we take $f=g$. If $R(0) \neq 0$, let $v$ be a principal monomial of $R$. For each $j, R=P(g+Y)=P_{j+1}\left(g-\left(u_{0}+\cdots+u_{j}\right)+Y\right)$. Since $P_{j+1}$ is unstable at $u_{j+1}$ and $g-\left(u_{0}+\cdots+u_{j}\right) \sim u_{j+1}$, it follows from $[6, \S 2.98 \mathrm{~L}]$ that the principal monomials of $R$ are $<u_{j+1}$. That is, $v<<u_{j} \forall j \geq$ 0 . Let $w \in K$ such that $R(w)=0$ and $w \sim v$. Then clearly, $g+w \approx \Sigma(\mathrm{u})$. We take $f=g+w$.

3.4. Corollary. If $\mathbf{u}$ is a critical chain for $P$ and each $u_{j}$ is a logaritbmic monomial, then $\exists$ an integer, say $p$, such that each $u_{j}$ bas logaritbmic rank $\leq p$ (cf. $[6, \$ 2.34]$ for terminology).

Proof. By $\$ 3.3, \exists f \in K$ such that $f \approx \Sigma(u)$. Since $Z$ is finitely enveloped, $f$ is contained in a $p$-constrained field (over $Z$ ) for some integer $p$. The corollary follows immediately.

3.5. Preliminary structure theorem. Let $\mathbf{u}=\left(u_{0}, u_{1}, \ldots\right)$ be a sequence into $K$ such that $P\left(u_{0}+\cdots+u_{j}\right)=0 \quad \forall j \geq 0$. Then it is impossible to bave $u_{j+1} \ll$ $u_{j} \forall j \geq 0$.

Proof. Assume the contrary. Then $\mathbf{u}$ is a critical chain for $P$. By $\$ 3.3$, If $\in K$ such that $P(f)=0$ and $f \approx \Sigma(u)$. Let $Q=P(f+Y)$. Define $v_{j}=u_{0}+\cdots$ $+u_{j}-f \forall j \geq 0$. Then $Q\left(v_{j}\right)=0 \forall j \geq 0$. Clearly, $v_{j} \sim-u_{j+1} \forall j \geq 0$. Thus, since 
$u_{j+1} \ll u_{j} \forall j \geq 0$, it follows that the set \{]$m[: m \in \operatorname{crit}(Q, \mathbf{Z})\}$ is an infinite set. This is in contradiction to the fact that, since $Q$ is first order, the set must be finite (cf. [1, p. 93, Remark (2)]).

IV. Proof of the abstract existence theorem. The proof falls into two cases: case one-Z is not algebraically closed; case two-Z is algebraically closed, but not first order closed. Similar developments are needed for each case. Case one requires the solution of an algebraic equation, while a first order a.d.e. is solved in case two. We give the proof for case two only, since it is, by far, the more difficult one.( 3 )

Outline of the proof for case two (cf. Introduction).

(1) $Q$ is selected and its properties described in $\$ \$ 4.1-10$.

(2) $\left(K^{\prime}, D^{\prime}\right)$ is constructed in $\$ \$ 4.10-13$.

(3) $\ll^{\prime}$ is constructed in $\$ \$ 4.14-21$.

(4) Asymptotic information about the operator $D^{\prime}$ is developed in $\$ \$ 4.22-29$.

(5) $Z^{\prime}=\left(\left(K^{\prime},<^{\prime}, U, C\right), D^{\prime}, \mathbf{x}\right)$ is show to be a GLF, finitely enveloped, and strongly attached to $Z$ in $\$ \$ 4.30-33$.

4.1. D. $n=\min \{s \in \pi: Z$ is not first order-(s+1)st degree closed\}.

4.2. D. A d.p. over $Z$, say $P$, is said to be critically unique (c.u.) iff (1) $\operatorname{card}(p m(P, Z))=1$, and (2) if $g \sim m \in p m(P, Z)$, then $\operatorname{card}(p m(P(g+Y), Z))$ $=1$, and, moreover, if $p \in \operatorname{crit}(P(g+Y), Z)$ and $p \ll m$, then $p \in p m(P(g+Y), Z)$.

4.3. General hypothesis for $\$ \$ 4.4-8$. $P$ is c.u.

4.4. Notation. The unique principal monomial of $P$ will be denoted by $c(P, 0)$, the unique principal monomial of $P(g+Y)$ will be denoted by $c(P, g)$.

4.5. L. Suppose $f, g \in K$ and $f g \neq 0$ and $g \sim c(P, 0)$. Then the d.p.'s, $P(g+Y)$ and $P(f Y)$ and $f P(Y)$, are c.u.

Proof. $P(g+Y)$ is c.u. by application of the second part of $\$ 4.2 . P(f Y)$ is c.u. by application of $\$ 2.20$ of [6]. The assertion about $f P(Y)$ is obvious.

4.6. Asymptotic alternative le mma. Let $g \sim b \sim c(P, 0)$. Then one of the following conditions bold: $c(P, g) \sim b+c(P, b)-g$ or $c(P, b) \sim g+c(P, g)-b$.

Proof. First we claim that either $c(P, g) \chi b-g$ or $c(P, b) \chi g-b$. Suppose the contrary. Then, by $[6, \S 2.95 \mathrm{~L}, \mathrm{D}], P(b+(g-b)) \ll P(b)$ and $P(g+(b-g)) \ll P(g)$. That is, $P(g) \ll P(b)$ and $P(b) \ll P(g)$, a contradiction.

By symmetry, it suffices to show that $c(P, g) \not b-g$ implies $c(P, b) \sim g+$ $c(P, g)-b$. There are three cases to consider.

(3) A proof for case one can be con structed by changing the results dealing with d.p.' $s$, given in IV, to corre sponding results dealing with polynomials. The latter results are uniformly less difficult. Reference must be made to [3] for information about the critical monomials of polynomials. 
Case one. $c(P, g) \gg b-g$. We have, by $[6, \S 2.95 \mathrm{~L}, \mathrm{D}], P(g+(b-g)) \sim$ $P(g)$. That is, $P(g) \sim P(b)$. Moreover, by the same reference, $P(b+(g+c(P, g)-b))$ $=P(g+c(P, g)) \ll P(g)$. Thus, we have $(a): P(b+(g+c(P, g)-b)) \ll P(b)$.

Suppose that $k \in K$ is $\ll c(P, g)$. Then $k+b-g \ll c(P, g)$. Thus, by [6, $\$ 2.95 \mathrm{~L}, \mathrm{D}], P(g+(k+b-g)) \sim P(g)$. That is, we have (b): $P(b+k) \sim P(b)$ whenever $k \ll c(P, g)$.

Consider $P(b+c(P, g))$. If $P(b+c(P, g)) \ll P(b)$, then (b) and [6, $\$ 2.95 \mathrm{~L}, \mathrm{D}]$ and the fact that $P$ is c.u. implies $c(P, g)=c(P, b)$. On the other hand, if $P(b+c(P, g)) \geq P(b)$, then (a) implies $c(P, g) \in \operatorname{crit}(P(b+Y), Z)$. Since $P$ is c.u. and $c(P, g) \ll c(P, 0)$, we have $c(P, g)=c(P, b)$. Thus, in either case, $c(P, g)$ $=c(P, b)$. Since $c(P, g) \gg b-g$, it follows that $c(P, b) \sim g+c(P, g)-b$.

Case two. $c(P, g) \approx b-g$, but $\chi b-g . P(g+(b-g)) \approx P(g)$ follows from $[6, \S 2.95 \mathrm{~L}, \mathrm{D}]$. That is, $P(b) \approx P(g)$. Moreover, as above, $P(b+(g+c(P, g)-b))$ $\ll P(g)$. Since $P(g) \approx P(b)$, we have $(c): P(b+(g+c(P, g)-b)) \ll P(b)$.

Since $P$ is c.u., $P(g+Y)$ is stable at $b-g$. Thus, if $k \ll b-g$, we have $P(g+(b-g)) \sim P(g+(b-g+k))$. That is, (d): $P(b) \sim P(b+k)$ whenever $k \ll$ $b-g$. We note that, since we are in Case two, $k \ll b-g$ iff $k \ll g+c(P, g)-b$.

Let $q \in M$ be $\sim g+c(P, g)-b$. If $P(b+q) \ll P(b)$, then $q=c(P, b)$ follows from (d) and the fact that $P$ is c.u.and $[6, \$ 2.95 \mathrm{~L}, \mathrm{D}]$. On the other hand, if $P(b+q) \geq P(b)$, then (c) implies $q \in$ crit $(P(g+Y), Z)$. Since $P$ is c.u. and $q$ $\ll c(P, 0)$, we have $q=c(P, b)$. Thus, $c(P, b) \sim g+c(P, g)-b$ in each case.

Case three. $c(P, g) \ll b-g$. Then $g+c(P, g)-b \sim g-b$. But, as before, $P(b+(g+c(P, g)-b)) \ll P(g)$ and $P(b+(g-b))=P(g)$. Thus, $P(b+Y)$ is unstable at $g-b$. Since $P$ is c.u. and $g-b \ll c(P, 0)$, it follows that $g-b \sim$ $c(P, b)$. Thus, $c(P, b) \sim g+c(P, g)-b$.

4.7. D. Let $\mathcal{F}$ be a set of d.p.'s over Z. Let $g$ be an element of $K$ and $\sim c(P, 0)$. Then $g$ is said to be a $P$-stable element for $\mathcal{F}$ iff $A(g+Y)$ is stable at $c(P, g)$ whenever $A \in \mathcal{F}$.

4.8. L. Let $\mathcal{F}$ be a finite, nonempty set of nonidentically zero d.p.'s which are either zero order or first order and of degree $\leq n$. Then 3 a P-stable element for $\mathfrak{F}$, say $g$.

Proof. The proof is by induction on $\operatorname{card}(\mathcal{F})$. Let $\mathcal{F}=\{A\}$. If $A$ is stable at $c(P, 0)$, we take $g=c(P, 0)$. If, on the other hand, $A$ is unstable at $c(P, 0)$, we may, since $\mathrm{Z}$ is first order-nth degree closed, select $g_{0} \sim c(P, 0)$ such that $A\left(g_{0}\right)=0$. If $A\left(g_{0}+Y\right)$ is stable at $c\left(P, g_{0}\right)$, we take $g=g_{0}$. If, on the other hand, $A\left(g_{0}+Y\right)$ is unstable at $c\left(P, g_{0}\right)$, we select $g_{1} \sim c\left(P, g_{0}\right)$ such that $A\left(g_{0}+g_{1}\right)=0$.

If the recursive procedure given above does not lead to a $R$-stable element 
for $\mathcal{F}, \exists$ an infinite sequence into $K$, say $\left(g_{0}, g_{1}, \cdots\right)$, such that $P\left(g_{0}+\cdots+g_{i}\right)=0$ and $g_{i+1} \ll g_{i} \forall i \geq 0$. But then the preliminary structure theorem of $\$ 3.5$ would be contradicted.

Let $q>1$ and suppose the lemma is true for $\operatorname{card}(F)<q$. Let $\mathcal{F}=$ $\left\{A_{1}, \cdots, A_{q}\right\}$. By the induction hypothesis, $\exists$ a $P$-stable element for $\mathcal{F}-\left\{A_{q}\right\}$, say $b_{0^{\circ}} \quad P\left(b_{0}+Y\right)$ is c.u. by $\$ 4.5$. Thus, we may apply the induction hypothesis to obtain a $P\left(b_{0}+Y\right)$-stable element for $\left\{A_{q}\left(b_{0}+Y\right)\right\}$, say $b_{1}$. It is easy to see that $g=b_{0}+b_{1}$ is a $P$-stable element for $\stackrel{q}{\mathscr{F}}$.

4.9. L. $\exists$ a first order, $(n+1)$ st degree d.p. over $Z$, say $P$, which is c.u. and, moreover, asymptotically nonsingular at $c(P, 0)$.

Proof. The proof is given in $\$ 4.34$.

4.10. General hypothes is for $\$ \$ 4.11-33 . Q$ is a first order, $(n+1)$ st degree d.p. over $Z$ which is c.u. and asymptotically nonsingular at $c(Q, 0)$ (w.1.o.g., we assume $c(Q, 0)=1)$. $z \in G$ is transcendental over $K$. $b \in G$ is such that $Q(z, b)$ $=0$. (We are making use of the fact that $Q=Q(Y, D Y)$ provides a polynomial over $K$ in two variables.)

4.11. L. $Q(z, Y)$ is irreducible over $K(z)$.

Proof. Suppose $Q(z, Y)$ is reducible over $K(z)$. Then, by Gauss' lemma, it is reducible over $K[z]$, say $Q(z, Y)=A(z, Y) B(z, Y)$. Thus, of course, $Q(Y, D Y)$ $=A(Y, D Y) B(Y, D Y)$. Since $Q$ is unstable at $c(Q, 0)$, it is easy to see (cf. $\$ 6.1$., also $\$ 4.14)$ that either $A(Y, D Y)$ or $B(Y, D Y)$ is unstable at $c(Q, 0)$. W.1.o.g., we assume that $A$ is unstable at $c(Q, 0)$. Since $A$ is a first order d.p. over $Z$ of degree $\leq n, A=0$ has a solution in $K, \sim c(Q, 0)$. But then $Q=0$ also has such a solution, contradicting the fact that it is c.u.

4.12. Corollary. Eacb element of $K(z, b)$ bas a unique representation of the form, $\Sigma\left\{a_{j} b^{j}: 0 \leq j \leq n\right\}$ where $a_{j} \in K(z)$, and also a representation of the form, $B(z, b)(A(z))^{-1}$ where $B$ and $A$ are polynomials over $K$ and the degree of $B(z, b)$ w.r.t. $b$ is $\leq n$.

4.13. D. Let $K^{\prime}$ denote the field, $K(z, b)$, and $D^{\prime}$, the derivative on $K^{\prime}$, defined by setting $D^{\prime} g=D g$, for $g \in K$, and $D^{\prime} z=b$.

4.14. Discussion. Let ] [ be the gauge function of $Z$ (cf. $[6, \$ 2.14 \mathrm{D}]$ ). Since each nonzero element of $K$ is $\sim$ to some element of $M$, ] [ is a valuation of $K$ with value group $U$ and, moreover, we have $f \ll g$ iff $] f[\ll] g[$ (cf. [3, Appendix, Note 1]). The above equivalence enables us indirectly to extend the asymptotic order $\ll$ to an asymptotic order for $K^{\prime}$, by extending ] [ to a valuation of $K^{\prime}$, say ] [', with value group $U$.

4.15. Notation ( $\$ \$ 4.16-29)$. The assertion " $f$ is an element of $K$ ' and equals 
$B(z, b)(A(z))^{-1}$ ", will mean, in addition to its usual meaning, that $B$ and $A$ are polynomials over $K$ and $B$ has degree $\leq n$ w.r.t. its second variable. If $R\left(Y_{1}, Y_{2}\right)$ is a polynomial over $K$, then the assertion " $g$ is a $Q$-stable element for $R$ " will mean that $g$ is a $Q$-stable element for $\{R(Y, \dot{D} Y)\}$. If $g$ is a $Q$-stable element (for some set of d.p.'s), $g+c(Q, g)$ will be denoted by $g^{*}$.

4.16. D. Suppose that $f$ is a nonzero element of $K^{\prime}$ and equals $B(z, b)(A(z))^{-1}$. Let $g$ be a $Q$-stable element for $\{A, B\}$. We define $] f^{\prime}=$ ] $B\left(g^{*}\right) A\left(g^{*}\right)^{-1}[$. We also define $] O^{\prime}=0$.

4.17. L. ] [' is a well-defined mapping and extends ] [.

Proof. There are two ambiguities in the definition of ] ['. Let $f \in K^{\prime}$ and $=B(z, b)(A(z))^{-1}$. Suppose that $g$ and $k$ are $Q$-stable elements for $\{A, B\}$. By \$4.6, we may assume, w.1.o.g., that $c(Q, g) \sim k^{*}-g$. Since $A(g+Y)$ is stable at $c(Q, g)$, we have $A\left(g^{*}\right) \sim A\left(g+\left(k^{*}-g\right)\right)=A\left(k^{*}\right)$. Therefore, $] A\left(g^{*}\right)[=] A\left(k^{*}\right)[$ and similarly for $B$. Thus, ] [' is well defined w.r.t. different choices of $Q$ stable elements.

It is easy to deal with the ambiguity due to the representation of $f$ as a quotient and clear that $] f[=] f\left[^{\prime}\right.$, for $f \in K$.

4.18. L. $] f+k\left[{ }^{\prime} \leq \max \{]\left[^{\prime},\right] k['\}\right.$, whenever $f, k \in K^{\prime}$.

Proof. Suppose that $f, k$ and $f+k$ are nonzero. Let $f=B(z, b)(A(z))^{-1}$ and $k=D(z, b)(C(z))^{-1}$. Let $g$ be a $Q$-stable element for $\{A, B, C, D, A C, C B+$ $A D\}$. Since ] [ is a valuation,

$$
] B\left(g^{*}\right) A\left(g^{*}\right)^{-1}+D\left(g^{*}\right) C\left(g^{*}\right)^{-1}\left[\leq \max \{] B\left(g^{*}\right) A\left(g^{*}\right)^{-1}[,] D\left(g^{*}\right) C\left(g^{*}\right)^{-1}[\}\right.
$$

The lemma follows.

If $f, k$ or $f+k=0$, the proof is trivial.

4.19. L. $] f k\left[{ }^{\prime}=\right] f[]^{\prime} k^{\prime}$, whenever $f, k \in K^{\prime}$.

Proof. Suppose that $f k \neq 0$ and $f=B(z, b)$ and $k=D(z, b)$. By Euclid's algorithm, 3 polynomials over $K$, say $F\left(Y_{1}\right), H\left(Y_{1}, Y_{2}\right)$, and $R\left(Y_{1}, Y_{2}\right)$, such that we have the polynomial equation, $F B D=H Q+R$, and $H$ and $R$ have degree $\leq n$ w.r.t. $Y_{2}$. Let $g$ be a $Q$-stable element for $\{F, B, D, H, R\}$. Consider the d.p. equation, $F B D(g+Y)=H Q(g+Y)+R(g+Y)$. By $\$ 6.1, F B D(g+Y)$ and $R(g+Y)$ are stable, while $H Q(g+Y)$ is unstable at $c(Q, g)$. Thus, $\$ 6.1$ implies ]FBD $\left(g^{*}\right)[=] R\left(g^{*}\right)\left[\right.$. Since ] [ is a valuation and $f k=R(z, b)(F(z))^{-1}$, we have ]$f k\left[{ }^{\prime}=\right] f\left[{ }^{\prime}\right]\left[{ }^{\prime} . H \neq 0\right.$ has been assumed w.o.l.g.

If $f=B(z, b)(A(z))^{-1}$ and $k=D(z, b)(C(z))^{-1}$, a slight change in the proof is necessary. If $f k=0$, the result is obvious. 
4.20. L. ] [' is a valuation of $K^{\prime}$ and has value group $U$.

Proof. By the definition of $]^{[}{ }^{\prime}$ and the two previous sections.

4.21. D. L. Whenever $f, g \in K^{\prime}$, we define $f<^{\prime} g$ iff $\left.] f^{\prime} \ll\right] g\left[{ }^{\prime}\right.$. The ordered quadruple $\mathrm{X}^{\prime}=\left(K^{\prime},<^{\prime}, U, C\right)$ is a graduated field $(G F)$. (The various asymptotic notions of $\mathrm{X}^{\prime}$ will be denoted by $]\left[{ }^{\prime},<^{\prime}, \sim^{\prime}, \approx^{\prime}\right.$, etc.) Moreover, if $k$ is a nonzero element of $K^{\prime}$, then $k \sim^{\prime} m$ for some $m \in M$.

Proof. $X^{\prime}$ is a GF by application of $\$ 4.20$ (cf. [3, Appendix, Note 1]).

Suppose that $k \in K^{\prime}-K$ and $=B(z, b)$. Let $g$ be a $Q$-stable element for $\{B\}$ and $B\left(g^{*}\right) \sim m \in M$. Let $f$ be a $Q$-stable element for $\{B, B-m\}$. Then $B\left(f^{*}\right)$ $\sim B\left(g^{*}\right)$. Thus, $B\left(f^{*}\right) \sim m$ or $B\left(f^{*}\right)-m \ll m$. Since $f$ is a $Q$-stable element for $\{B-m\}$, it follows that $k \sim^{\prime} m$.

If $k$ is of the form, $B(z, b)(A(z))^{-1}$, a slight change in the above proof is necessary. If $k \in K$, the result is obvious.

4.22. General hypothesis for $\$ \S 4.23-29 . r \in \Re . g$, an element of $K$, is 1. $z^{\prime}=(z-g)(c(Q, g))^{-1}$.

4.23. L. Suppose that $Q$ is r-inscribed. Let $Q$, be the polynomial over $K$ such that $Q_{r}\left(Y, D_{r} Y\right)=Q(Y)\left(D_{r} Y \equiv x_{0} x_{1} \cdots x_{r} D Y\right)$. Let $Q_{r}(z, Y)=\Sigma q_{i} Y^{i}$. Then $q_{0} \ll<^{\prime} q_{1}$ and $q_{i} \preceq^{\prime} q_{1} \forall i$.

Proof. Let $H$ be the polynomial over $K$ such that $H\left(Y, D_{r} Y\right)=Q_{r}\left(1+Y, D_{r} Y\right)$ $=\Sigma b_{i j} Y^{i}\left(D_{r} Y\right)^{j} . A$ and $B$ will refer, respectively, to the d.'.'s, $\Sigma\left(b_{i 0} Y^{i}: i \geq 0\right\}$ and $\Sigma\left(b_{i j} Y^{i}\left(D_{r} Y\right)^{j}: j \geq 1\right\}$. Since $Q$ is asymptotically nonsingular at $1(=c(Q, 0))$, we have $b_{i j} \preceq b_{01} \forall j \geq 1$ by $[6, \S 3.9 L]$. Thus, clearly, $B$ is stable at all nonzero elements of $K$ which are $\ll 1$. Indeed, for such an element $f, B(f) \sim$ $b_{01} D_{r} f$

Let $k$ be a $Q(1+Y)$-stable element for $\{A\}$. We have $Q(1+k+Y)=$ $A(k+Y)+B(k+Y) \cdot Q(1+k+Y)$ is unstable, but $A(k+Y)$ and $B(k+Y)$ are stable at $c(Q, 1+k)$. Thus, by $\$ 6.1] A,(k+c(Q, 1+k))[=] B(k+c(Q, 1+k))[$. By the stability property of $B$ given above, we see that $A(k+c(Q, 1+k))<<$ $b_{01} \cdot q_{0}=A(z-1)$ by definition. Clearly, $1+k$ is a $Q$-stable element for $\{A(Y-1)\}$. Therefore, $] A(k+c(Q, 1+k))[=] q_{0}\left[{ }^{\prime}\right.$. Thus $q_{0} \ll<^{\prime} b_{01}$ '

$q_{1}=\Sigma\left\{b_{i 1}(z-1)^{i}: i \geq 0\right\}$. Since $z-1 \ll<^{\prime} 1, q_{1} \sim^{\prime} b_{01}$. Thus, $q_{i} \varliminf^{\prime} q_{1}$ follows from the condition, $b_{01} \geq^{\prime} b_{i j}$ for $j \geq 1$.

4.24. Corollary. Let $Q^{\prime}(Y)=Q(g+c(Q, g) Y)$. Suppose that $Q^{\prime}$ is r-inscribed. Let $Q_{r}^{\prime}$ be the polynomial over $K$ such that $Q_{r}^{\prime}\left(Y, D_{r} Y\right)=Q^{\prime}(Y)$. If $Q_{r}^{\prime}\left(z^{\prime}, Y\right)=$ $\Sigma c_{i} Y^{i}$, then $c_{0} \ll{ }^{\prime} c_{1}$ and $c_{i} \varliminf^{\prime} c_{1} \forall i$. 
Proof. The proof is analogous to that of $\$ 4.23$ with $Q^{\prime}$ and $z^{\prime}$ replacing $Q$ and $z$, respectively.

4.25. L. $D_{r}^{\prime} z$ and $D_{r}^{\prime} z^{\prime}$ are $\ll<^{\prime} 1$.

Proof. $D_{r}^{\prime} z=x_{0} x_{1} \cdots x_{r} b$. Clearly, 1 is a $Q$-stable element for $\left\{D_{r} Y\right\}$. Thus, by definition, $] D_{r}^{\prime} z\left[^{\prime}=1 D_{r}(1+c(Q, 1))\left[=D_{r}(c(Q, 1))\left[\right.\right.\right.$. But, $D_{r}(c(Q, 1)) \ll 1$ since $c(Q, 1) \ll 1$. Thus $D_{r}^{\prime} z \ll<^{\prime} 1$.

Consider $D_{r}^{\prime} z^{\prime}$. Clearly, $g+c(Q, g)$ is a $Q$-stable element for

$$
\left\{D_{r}\left((Y-g)(c(Q, g))^{-1}\right)\right\} \text {. }
$$

Thus, by definition, $\left.] D_{r}^{\prime} z{ }^{\prime}=\right] D_{r} p\left[\right.$ where $p=c(Q, g+c(Q, g))(c(Q, g))^{-1}$. Since $p \ll 1, D_{r} p \ll 1$. Thus, $D_{r}^{\prime} z^{\prime} \ll<^{\prime} 1$.

4.26. L. Suppose $k$ is an element of $K^{\prime}$ which is $\approx^{\prime} 1$ and equals $A(z)$. Then $D_{r}^{\prime} k \ll<^{\prime} 1$.

Proof. Let $f$ be a $Q$-stable element for $\{A\}$. Then $A\left(f^{*}\right) \approx 1$. Let $B(Y)=$ $\Sigma b_{i} Y^{i}=A(f+c(Q, f)(1+Y))$. By a straightforward application of [3, 23$]$, we have $b_{i} \preceq b_{0} \forall i . b_{0}=A\left(f^{*}\right) \approx 1$. Let $z^{\prime \prime}=(z-f)(c(Q, f))^{-1}$. Then $A(z)=$ $B\left(z^{\prime \prime}-1\right)$. Thus, $D_{r}^{\prime} k=\sum\left\{i b_{i}\left(z^{\prime \prime}-1\right)^{i-1} D_{r}^{\prime} z^{\prime \prime}+\left(z^{\prime \prime}-1\right)^{i} D_{r} b_{i}\right\} . \quad D_{r} b_{i} \ll 1$ since $b_{i} \preceq 1$. $D_{r}^{\prime} z^{\prime \prime} \ll<^{\prime} 1$ by $\$ 4.25$. Thus, since $z^{\prime \prime}-1 \ll<^{\prime} 1$, all the terms in the sum for $D_{r}^{\prime} k$ are $<^{\prime} 1$. Therefore, $D_{r}^{\prime} k \ll<^{\prime} 1$.

4.27. L. For $r$ sufficiently large $\left(D_{r}^{\prime}\right)^{2} z$ and $\left(D_{r}^{\prime}\right)^{2} z^{\prime}$ are $<^{\prime} 1$.

Proof. Let $r$ be so large that $Q$ is $r$-inscribed. By $\$ 4.23$ and its notation, if $Q_{r}(z, Y)=\Sigma q_{i} Y^{i}$, then $q_{0} \ll^{\prime} q_{1}$ and $q_{i} \preceq^{\prime} q_{1} \forall i$. W.1.o.g., we may assume $q_{1} \sim^{\prime} 1$. Then $\left(D_{r}^{\prime}\right)^{2} z<<^{\prime} 1$ results from differentiating the equation, $\Sigma q_{i}\left(D_{r}^{\prime} z\right)^{i}$ $=0$, and using the estimates, $D_{r}^{\prime} z$ and $D_{r}^{\prime} q_{i} \ll{ }^{\prime} 1$ (cf. $\$ \$ 4.25-26$ ).

The estimate of $\left(D_{r}^{\prime}\right)^{2} z^{\prime}$ follows in an analogous way by considering the d.p., $Q(g+c(Q, g) Y)$, and $\$ 4.24$.

4.28. L. Suppose $k$ is an element of $K^{\prime}$ which $\approx^{\prime} 1$ and equals $B(z, b)$. Then $D_{r}^{\prime} k \ll<1$.

Proof. Let $f$ be a $Q$-stable element for $\{B\}$ and $z^{\prime \prime}=(z-f)(c(Q, f))^{-1}$. Let $t$ be an integer such that $B(f+c(Q, f) Y)$ and $Q(f+c(Q, f) Y)$ are $t$-inscribed and $\left(D_{t}^{\prime}\right)^{2} z^{\prime \prime} \ll<^{\prime}$ 1. Let $B(f+c(Q, f)(1+Y))=\Sigma b_{i j} Y^{i}\left(D_{t} Y\right)^{j}$. By a straightforward application of $[6, \S 2.99 \mathrm{~L}], h_{i j} \leq h_{00} \forall i, j . h_{00}=B\left(f^{*}\right) \approx 1 . k=$ $\Sigma b_{i j}\left(z^{\prime \prime}-1\right)^{i}\left(D_{t}^{\prime} z^{\prime \prime}\right)^{j} . D_{t}^{\prime} k<<^{\prime} 1$ follows by differentiating the preceding equation and recalling the estimates, $b_{i j} \preceq 1, z^{\prime \prime}-1<<^{\prime} 1, D_{t}^{\prime} z^{\prime \prime}<<^{\prime} 1$, and $\left(D_{t}^{\prime}\right)^{2} z^{\prime \prime} \ll<^{\prime} 1$. 
If $r \geq t$, the above proof applies. On the other hand, if $r<t$, then $D_{r}^{\prime} k \ll<^{\prime}$ $D_{t}^{\prime} k \ll<1$.

4.29. L. Suppose $k$ is an element of $K^{\prime}$ which is $\approx^{\prime} 1$. Then $D_{r}^{\prime} k \ll<^{\prime} 1$.

Proof. We may write $k=B(z, b)(A(z))^{-1}$. W.1.o.g., we may assume (cf. $\$ 4.21$ ) $B(z, b) \approx{ }^{\prime} A(z) \approx{ }^{\prime} 1$. The result now follows by differentiating the above equation and considering $\$ 4.26$ and $\$ 4.28$.

4.30. L. $\mathbf{Z}^{\prime}=\left(\left(K^{\prime},<^{\prime}, U, C\right), D^{\prime}, \mathbf{x}\right)$ is a $G L F$.

Proof. By $\$ 4.21$ and the definition of a GLF, it remains only to show that $D^{\prime}$ is stable, in the context of $Z^{\prime}$, at the nonconstant logarithmic monomials. But, by $\$ 6.2$, it suffices to show what has been shown in $\$ 4.29$.

4.31. D. A d.p. which is c.u., say $P$, is said to be rank-stable iff (1) whenever $P$ is $r$-inscribed, then $c(P, 0) \in M_{r}$, and (2) whenever $g \sim c(P, 0)$ and $P(g+Y)$ is $q$-inscribed, then $c(P, g) \in M_{q}$.

In general, when a d.p., say $R$, is $r$-inscribed and $p \in \operatorname{crit}(R, Z)$, but $\notin M_{r}$, we will say that a rank rise occurs at $p$.

4.32. L. $\exists$ nonzero elements of $K$, say $a, b$, and $d$, such that $b \sim c(Q, 0)$ and $a Q(b+d Y)$ is rank-stable.

Proof. The proof is given in $\$ 4.35$.

4.33. L. $Z^{\prime}$ is strongly attacbed to $\mathbf{Z}$ and $K^{\prime} \subset G$.

Proof. It only remains to show that $Z^{\prime}$ is finitely enveloped. Let $\mathfrak{F}=$ $\left\{f_{1}, f_{2}, \cdots, f_{p}\right\}$ be a finite subset of $K^{\prime}$. We must show that $\mathcal{F}$ is contained in a field which is constrained over $\mathbf{Z}^{\prime}$. W.1.o.g., we may assume that, $\forall i, f_{i}=$ $B_{i}(z, b)$ where $B_{i}$ is a polynomial over $K$. Let $a, b$, and $d$ be elements of $K$ such that $L=a Q(b+d Y)$ is rank-stable (cf. $\$ 4.32)$. Since $Z$ is finitely enveloped, $\exists r \in \pi$ and an $r$-constrained field (over $Z$ ), say $H$, which contains $\{a, b, d\}$ and the coefficients of both $L$ and $B_{i}, \forall i$. Since $Z$ is algebraically closed (cf. $\$ 1.7$ ), we may assume (cf..[6, $\$ 2114 \mathrm{~L}])$ that $H$ is algebraically closed.

Let $z^{\prime \prime}=(z-b) d^{-1}$. Let $H^{\prime}$ be the differential field $H\left(z^{\prime \prime} D^{\prime} z^{\prime \prime}\right)$. Since $H^{\prime}$ obviously contains $\mathcal{F}$, it suffices to show that each nonzero element of $H^{\prime}$ is $\sim^{\prime}$ to a logarithmic monomial of rank $\leq r$. Or, what is the same, that ] [' (restricted to $H^{\prime}$ ) is a valuation of $H^{\prime}$ with value group $U_{r}$.

First we consider $H\left(z^{\prime \prime}\right)$. It suffices to consider a polynomial over $H$ in $z^{\prime \prime}$, say $A\left(z^{\prime \prime}\right)$. Since $L$ is rank-stable and, moreover, $H$ is algebraically closed, $\exists$, by an examination of the proof of $\$ 4.8$, an element of $H$, say $s$, which is an $L$ stable element for $\{A\}$. By a straightforward argument (cf. $[6, \S 2.20 \mathrm{~L}]$ ), we have 
$c(Q, b+d s) \sim d c(L, s)$. Thus, it follows easily that $b+d s$ is a $Q$-stable element for $\left\{A\left((Y-b) d^{-1}\right)\right\}$. Therefore, $] A\left(z^{\prime \prime}\right)\left[{ }^{\prime}=\right] A\left(s+d^{-1} c(Q, b+d s)\right)[$. But, since, clearly, $s+d^{-1} c(Q, b+d s) \in H$, we have $A\left(s+d^{-1} c(Q, b+d s)\right) \in H$. Thus, $A\left(z^{\prime \prime}\right)$ is $\sim^{\prime}$ to a logarithmic monomial of rank $\leq r$ (cf. $\$ 4.21$ ). The same is true for all nonzero elements of $H\left(z^{\prime \prime}\right)$. Thus, ] [' (restricted to $H\left(z^{\prime \prime}\right)$ ) is a valuation of $H\left(z^{\prime \prime}\right)$ with value group $U_{r}$.

Since $H^{\prime}$ is algebraic over $H\left(z^{n}\right)$ and $U_{r}$ cannot be enlarged by adding roots of its own elements, it follows from valuation theory considerations (cf. [2, Corollary $1, \mathrm{p} .300]$ ) that ] [ (restricted to $H^{\prime}$ ) is a valuation of $H^{\prime}$ with value group group. $U_{r}$.

4.34. Proof of $\$ 4.9$. Since $Z$ is first order-nth degree closed, but not first order- $(n+1)$ st degree closed, $\exists$ a first order d.p. over $Z$, say $P$, of degree $n+1$ and $m \in \operatorname{crit}(P, Z)$ such that $P=0$ has no solution, $\sim m$, in $K$. Let $\mathbb{Q}$ denote the set of all ordered pairs, $(P, m)$, which satisfy the above conditions. We claim that $\mathcal{Q}$ contains an element, say $(Q, p)$, such that $Q$ is asymptotically nonsingular at $p$. Indeed, in the contrary case, a contradiction can be obtained by a straightforward use (cf. $\$ 1.5$ and $\$ 3.2$ for similar applications) of Strodt's structure theorem (cf. $[6, \$ 6.10]$ ).

Let $(Q, p)$ be as claimed. By $[6, \S 3.35], \exists a, b, d \in K$ such that $b \sim p$ and $L=a Q(b+d Y)$ is quasilinear (cf. $[6, \$ 4.6])$. The theory of quasilinear d.p.'s is thoroughly developed in $[6$, Chapter 4$]$. By $[6, \$ 4.13], L$ has a unique principal monomial, say $t$. Moreover, if $f \sim t$, then, by $[6, \S 4.20], T(f, L)$ $\left(\equiv(\partial L(f) / \partial Y)^{-1} L(f+Y)\right)$ is also quasilinear and, thus, it has a unique principal monomial. Therefore, by definition, $L$ is c.u. if we can show that: whenever $g \sim t$ and $r \in \operatorname{crit}(L(g+Y), Z)$ is $\ll t$, then $r$ is the principal monomial of $L(g+Y)$.

Suppose the contrary for a particular $g$ and $r$. In this case we can show (cf. [7, Lemma 45, p. 104]) that $\dot{w}(Y)$, the homogeneous linear part of $T(g, L)$, is unstable at $r$. We claim that $T(\equiv T(g, L))$ is c.u. Suppose the contrary. Let $t_{0}$ be the principal monomial of $T$. Then $\exists k \sim t_{0}$ and $s \in \mathrm{crit}(T, \mathrm{Z}) \ll t_{0}$, such that $s$ is not the principal monomial of $T(k+Y)$. Then, as before, $\dot{w}_{k}(Y)$, the homogeneous linear part of $T(k, T)$, is unstable at $s$. It can be shown (cf. [7, Lemma 44, p. 102]) that $r \approx s$ follows from $r \in \operatorname{crit}(\dot{w}(Y), Z)$ and $s \in \operatorname{crit}\left(\dot{w}_{k}(Y), Z\right)$. This contradicts $s \ll r$.

4.35. Proof of $\$ 4.32$. We may assume, w.l.o.g., that $Q$ is quasilinear since, in the contrary case, $\exists a, b, d \in K$ such that $a Q(b+d Y)$ is quasilinear (cf. [6, \$3.35]).

If $H$ is quasilinear then no rank rise can occur at its principal monomial, except under very special conditions (cf. $[6, \S 4.24]$ ). One can prove (cf. [7, Lemma 
52, p. 111]) the following:

Lemma a. If $H$ is quasilinear and c.u. and a rank rise occurs at $c(H, 0)$, then $T(c(H, 0), H)$ is rank-stable.

Suppose that $Q$ is not rank-stable. $Q$ can fail in two ways. First, a rank rise may occur at $c(Q, 0)$. Then, by Lemma a, $T(c(Q, 0), Q)$ is rank-stable. Second, $Q(k+y)$ may have a rank rise at $c(Q, k)$ for some $k \sim c(Q, 0)$. Clearly, $T(k, Q)$ also has a rank rise at $c(Q, k)$. Thus, by Lemma a, $T(c(Q, k), T(k, Q))$ is rank-stable. Therefore, in either case, a d.p. of the form, $a Q(b+d Y)$, with $b \sim c(Q, 0)$, is shown to be rank-stable.

\section{Applications.}

General hypothesis for $V_{0} Z=((K, \ll, U, C), D, \mathbf{x})$ is a GLF (not necessarily finitely enveloped) and $C$ is algebraically closed. $P$ is a first order d.p. which is inscribed over $\mathrm{Z}$ (cf. $[6, \$ 2.106 \mathrm{D}]$ ).

First order structure the orem. Suppose that $P=0$ bas an infinite sequence of adjoinable solutions, say $v_{0}, v_{0}+v_{1}, \cdots, v_{0}+v_{1}+\cdots+v_{n}, \cdots$. (By this we mean that for some field, say $H$, which is constrained over $\mathrm{Z}$ and contains the coefficients of $P, \exists$ a nondecreasing sequence of constrained overfields of $H_{\text {, say }} H_{0}, H_{1}, \cdots$, such that $v_{i} \in H_{i} \forall i$.) Then it is impossible to bave $v_{i+1} \ll$ $v_{i} \forall i$.

Proof. Suppose the contrary. We will obtain a contradiction by embedding that part of $\mathrm{Z}$, which is relevant to $P$ and the $v_{i}$, in a first order closed GLF and applying the preliminary structure theorem of $\$ 3.5$.

Let $G=\bigcup\left\{H_{i}\right\}$. Let $M(U)$ be the set of (unit) logarithmic monomials of $\mathbf{Z}$. Let $F=G\langle M\rangle$, the differential field generated over $G$ by $M$. Let $\mathbf{Z}_{0}=$ $\left(\left(F,<^{\prime}, U, C\right), D^{\prime}, \mathbf{x}\right)$, where $\ll^{\prime}$ and $D^{\prime}$ refer, respectively, to the restrictions of $\ll$ and $D$ to $F$. It is easy to see that $Z_{0}$ is a finitely enveloped GLF. Let $\mathbf{Z}_{0}^{*}$ be a first order differential closure of $\mathbf{Z}_{0}$. The preliminary structure theorem is then contradicted by $P$ and the $v_{i}$.

Example 1 (due to E. R. Kolchin). The nonalgebraic, first order differential equation, $x \log (x)(d U / d x)-U \log (U)=0$, where $U=x+(1-x) Y$, has among its solutions, the functions, $1,1+x^{-1}, 1+x^{-1}+x^{-2}, \ldots$ and so on.

By the first order structure theorem, a chain of solutions, such as $1,1+$ $x^{-1}, 1+x^{-1}+x^{-2}, \cdots$, is impossible for an algebraic, first order differential equation. Of course, much more general chains of solutions than $1,1+x^{-1}, \ldots$ are denied to algebraic, first order differential equations by the same theorem.

Example 2 (due to W. Strodt). The algebraic, second order differential equation, $U(d U / d x)+x U_{d x}^{d}(d U / d x)-x(d U / d x)^{2}=0$, where $U=x+(1-x) Y$, has 
among its solutions the functions, $1,1+x^{-1}, 1+x^{-1}+x^{-2}, \cdots$ and so on.

This shows that a general structure theorem is unavailable (4) for algebraic differential equations of order greater than one.

A result on critical chains. Let $\mathrm{m}=\left(m_{0}, m_{1}, m_{2}, \ldots\right)$ be a critical chain for $P$ (cf. $\$ 3.1$ ), where, for each $i, m_{i}$ is a logarithmic monomial. Then $\exists$ a nonnegative integer $p$, such that, for each $i$, the logarithmic rank of $m_{i}$ is $\leq p$.

Proof. Let $H$ be a constrained field over $\mathrm{Z}$ which contains the coefficients of $P$. Let $F=H\langle M\rangle$. Define $\mathrm{Z}_{0}$ and $\mathrm{Z}_{0}^{*}$ as in the previous proof. The result now follows from $\$ 3.4$ provided that $m$ is a critical chain for $P$ w.r.t. $Z_{0^{*}}^{*}$ But this is clear since the fact that $m$ is a critical chain for $P$ w.r.t. $\mathbf{Z}$ depends only on the value of $P$ at the points of $F$ (cf. $[6, \$ 2.97 \mathrm{~L}]$ ) and $F$ and the asymptotic ordering of its elements is common to $\mathbf{Z}, \mathbf{Z}_{0}$, and $\mathbf{Z}_{0^{*}}^{*}$

Example 3 (Strodt). The differential polynomial, $P(Y)=4 Y-x^{2}(d Y / d x)^{2}-$ $x d Y / d x$, has a critical chain, $\left(m_{0}, m_{1}, \cdots\right)$, where $m_{0}=(\log x)^{2}$ and $m_{1}=$ $-2(\log x)(\log (\log x))$ and the higher elements in the chain are monomials of rank $\leq 2$. These facts can be verified with the aid of Bank's algorithm.

The coefficient domain for $P$ has rank 0 , i.e., the coefficients involve only constants and powers of $x$. The critical chain has rank 2. Thus, we say the rank rise here is 2. This is the largest rank rise which has been observed in an example. The maximum rank rise question is an open problem. However, certain bounds have been obtained (unpublished) by Strodt.

VI. Appendix.

6.1. Let $F$ be a field of characteristic zero with a valuation, say $V$ (cf. $[2, \mathrm{pp} .296-300])$. Let $\ll$ be the ordering of the value group of $V . g$ is a nonzero element of $F$.

Definition. If $f, b \in F$, then $f \sim b$ means $V(f-b) \ll V(b)$. If $A$ is an operator on $F$, then $A$ is said to be stable at $g$ iff $b \sim g$ implies $A(b) \sim A(g)$.

Lemma. Let $A, B$ and $C$ be operators on $F$ (1) If $A$ and $B$ are stable at g, so is $A B$. ( $B y A B$ we mean the operator $A B: f \mapsto A(f) B(f)$.) (2) If $A$ is stable and $B$ is unstable, then $A B$ is unstable at $g$. (3) If $A=B+C$ and $A, C$ are stable at $g$ and $B$ is unstable at $g$, then $V(A(g))=V(C(g))$. (4) If $A=B+$ $C$, and $A$ is unstable at $g$, but $B$ and $C$ are stable at g, then $V(B(g))=V(C(g))$.

Proof. (1) ((2)) follows from the easily established fact that $f \sim s$ and $d \sim$ $(\chi) t$ implies $f d \sim(\chi)$ st.

Consider (3). It clearly suffices to show that $V(B(h))<V(C(b))$ whenever $h \sim g$.

(4) However, certain structure theorems can be proved for broad classes of higher order al gebraic differential equations (cf. [7, Theorem 14, p. 202]). 
Suppose to the contrary that $\exists$ an $h_{0} \sim g$ such that $\left.V\left(B\left(h_{0}\right)\right) \gg V C\left(h_{0}\right)\right)$. Clearly, $B$ is unstable at $b_{0}$ since it is unstable at $g$ and $b_{0} \sim g$. Thus, $\exists$ an $b_{1} \sim b_{0}$ such that $V\left(B\left(b_{1}\right)-B\left(b_{0}\right)\right) \geqq V\left(B\left(b_{0}\right)\right)$. On the other hand, since $C$ is stable at $g$, it is also stable at $b_{0}$. Thus, $V\left(C\left(b_{1}\right)-C\left(b_{0}\right)\right) \ll V\left(C\left(b_{0}\right)\right)$. Combining inequalities, we have $V\left(C\left(b_{1}\right)-C\left(b_{0}\right)\right) \ll V\left(B\left(b_{1}\right)-B\left(b_{0}\right)\right)$. It follows by an easy valuation argument that $V\left(A\left(b_{1}\right)-A\left(b_{0}\right)\right)=V\left(B\left(b_{1}\right)-B\left(b_{0}\right)\right)$. Since $A$ is clearly stable at $b_{0}, V\left(A\left(b_{1}\right)-A\left(b_{0}\right)\right)<<\left(A\left(b_{0}\right)\right)$. Thus, by collecting equalities and inequalities, we have $V\left(A\left(b_{0}\right)\right) \gg V\left(B\left(b_{0}\right)\right) \geqq V\left(C\left(b_{0}\right)\right)$. That is, $V\left(A\left(b_{0}\right)\right)$ $\gg \max \left\{V\left(B\left(b_{0}\right)\right), V\left(C\left(b_{0}\right)\right)\right\}$. This is a contradiction since $A=B+C$ and $V$ is a valuation.

(4) can be proved by similar considerations.

6.2. Lemma. Let $\mathrm{Z}=((K, \ll, U, C), D, \mathrm{x})$ satisfy all the defining conditions for a GLF (cf. $[6, \S 2.4])$, except possibly condition (2) of $[6, \$ 2.4]$, i.e., we do not assume that $D$ is stable at each element of $U-\{1\}$. We do assume that $U$ consists only of logarithmic monomials. The following conditions are equivalent.

(1) $D$ is stable at eacb element of $U-\{1\}$.

(2) $D_{r} f \ll 1$, whenever $f \ll 1$ and $r \in \pi$.

(3) $D_{r} b \ll 1$, whenever $b \approx 1$ and $r \in \Re$.

Proof. (2) and (3) are easily seen to be equivalent. Moreover, if (1) holds then $Z$ is a GLF and $[6, \S 2.31 \mathrm{~L}]$ implies (2) and, thus, (3) also.

It suffices to show that (2) implies (1). Let $u \in U-\{1\}$. If $b \sim u$, then $b=u+u e$ with $e \ll 1$. $D b=D u+u D e+e D u$. Thus, $D b \sim D u$, provided $u D e$ $\ll D u$ or, in other words, provided $u(D u)^{-1} D e \ll 1$. We claim that $u(D u)^{-1} \approx$ $x_{0} x_{1} \cdots x_{r}$ for some $r \in \Re$. Of course, if our claim is true, (2) implies $u(D u)^{-1} D e$ $\ll 1$ and, thus, $D u \sim D b$.

Let us prove our claim. Since $u \neq 1$, it has the form, $x_{s}^{a} x_{s+1}^{b} \cdots x_{j}^{t}$ with $a \neq 0$. By differentiation, $u^{-1} D u=a\left(x_{0} x_{1} \cdots x_{s}\right)^{-1}+\cdots+t\left(x_{0} x_{1} \cdots x_{j}\right)^{-1}$. If we can show that $x_{n} \gg 1 \forall n \in \Re$, it will follow that $u^{-1} D u \approx\left(x_{0} x_{1} \cdots x_{s}\right)^{-1}$ and, thus, $u(D u)^{-1} \approx_{x_{0} x_{1}} \cdots x_{s}$. We have $x_{n} \neq 1$, for in the contrary case the condition $D_{n-1}\left(x_{n}\right)=1$ is contradicted. Since $x_{n} \in U$, it is either $\gg$ or $\ll 1$. But, if $x_{n} \ll 1$, then (2) implies $D_{n-1}\left(x_{n}\right) \ll 1$ which contradicts $D_{n-1}\left(x_{n}\right)=1$. Thus, $x_{n} \gg 1 \forall n \in \Re$.

\section{BIBLIOGRAPHY}

1. S. Bank, On the instabliity theory of differential polynomials, Ann. Mat. Pura Appl. (4) 74 (1966), 83-111. MR 34 \#4623.

2. S. Lang, Algebra, Addison-Wesley, Reading, Mass., 1965. MR 33 \#5416.

3. W. Strodt, On the algebraic elosure of certain partially ordered fields, Trans. Amer. Math. Soc. 105 (1962), 229-250. MR 25 \#3934. 
4. W. Strodt, Graduated logarithmic fields and stability, Univ. of Wisconsin, U.S. Army MRC Tech. Summary Rep. No. 489, June, 1964

5. - Asymptotic expansions and structure theorems, Preliminary report, Notices Amer. Math. Soc. 18 (1971), 370-371. Abstract \#683-B27.

6. W. Strodt and R. K. Wright, Asymptotic behavior of solutions and adjunction fields for nonlinear first order differential equations, Mem. Amer. Math. Soc. No. 109 (1971), 284 pp. MR 44 \#1884.

7. J. Turcheck, Contributions to the abstract theory of asymptotic expansions, for solutions of algebraic differential equations, Dissertation, Columbia University, New York, N. Y., 1972.

DEPARTMENT OF MATHEMATICS, ST. LATRENCE UNIVERSITY, CANTON, NEW YORK 13617 